\title{
Characterizing the object categories two children see and interact with in a dense dataset of naturalistic visual experience
}

\author{
Bria Long ${ }^{1}$, George Kachergis ${ }^{1}$, Naiti Bhatt ${ }^{2}$, Michael C. Frank ${ }^{1}$ \\ bria@stanford.edu, kachergis@stanford.edu,nbhatt@hmc.edu, mcfrank@stanford.edu \\ ${ }^{1}$ Department of Psychology, Stanford University \\ ${ }^{2}$ Scripps College
}

\begin{abstract}
What do infants and young children tend to see in their everyday lives? Relatively little work has examined the categories and objects that tend to be in the infant view during everyday experience, despite the fact that this knowledge is central to theories of category learning. Here, we analyzed the prevalence of the categories (e.g., people, animals, food) in the infant view in a longitudinal dataset of egocentric infant visual experience. Overall, we found a surprising amount of consistency in the broad characteristics of children's visual environment across individuals and across developmental time, in contrast to prior work examining the changing nature of the social signals in the infant view. In addition, we analyzed the distribution and identity of the categories that children tended to touch and interact with in this dataset, generalizing previous findings that these objects tended to be distributed in a Zipfian manner. Taken together, these findings take a first step towards characterizing infants' changing visual environment, and call for future work to examine the generalizability of these results and to link them to learning outcomes.
\end{abstract}

Keywords: Object categorization; infant visual experience; head-mounted cameras; longitudinal data.

\section{Introduction}

What do children tend to see in their everyday lives? While an understanding of children's visual environment is central to both theories of language acquisition and visual development, we know remarkably little about the categories and objects that tend to be in the infant view, or in what format they are experienced. For example, how often do infants tend to see animals in real-life vs. in storybooks or as toys? How consistent are children's visual environments across individuals and across developmental time?

Over the past decade, researchers have begun to answer these questions by documenting the infant egocentric perspective using head-mounted cameras (Franchak, Kretch, Soska, \& Adolph, 2011; Yoshida \& Smith, 2008) and quantifying the degree to which there are substantial shifts in infants' viewpoints that may have downstream developmental consequences. As adults, it is hard to intuit how strange this viewpoint can be, and how much it varies across development, transitioning over the first two years of life from closeup views of faces to restricted views of hands manipulating objects (Fausey, Jayaraman, \& Smith, 2016; Long, Kachergis, Agrawal, \& Frank, 2020), with children's postural developments to a large extent shaping what they see (Sanchez, Long, Kraus, \& Frank, 2018). Most work, however, has focused on documenting the social information that infants and children have access to across early development (Fausey et al., 2016; Sanchez et al., 2018; Yoshida \& Smith, 2008).

More recent research has made progress towards understanding what objects tend to be the infant view, starting with analyzing the basic-level categories (e.g., spoons, cups) in the view of 8-10 month-olds during mealtime. This work suggests that a small number of objects are both pervasively present during mealtime and among infants' first-learned words (Clerkin, Hart, Rehg, Yu, \& Smith, 2017), pointing towards a link between visual experience and early word and category learning.

Thus, a more complete understanding of the visual environment of infants and young children could yield insights about the inputs to both category learning and word learning. Indeed, different distributions of these visual referents lead to constraints on the kinds of learning mechanisms that must operate to form robust category representations - and to learn words for these categories. However, at present, no datasets are sufficiently annotated to constrain these theoretical accounts.

For example, if the categories in the infant view shift dramatically over the first few years of life, then we might expect infants to learn about certain categories earlier vs. later during development. Prior work documenting the proportion of social information in view has suggested that children see more hands relative to faces in this same age range (Fausey et al., 2016; Long et al., 2020). Thus, one possibility is that as children learn to crawl and walk (Franchak et al., 2011; Long et al., 2020; Sanchez et al., 2018), the categories that children are likely to interact with (i.e., toys, small objects) may also become more prevalent in the child's view. If this was the case, this finding would support a view where the inputs to early category learning are shaped by children's own ability to actively explore their environment.

On the other hand, the broad characteristics of children's visual environments may be relatively stable and mostly determined by the activities that they tend to engage in. Indeed, some theoretical accounts have suggested that the statistics of children's visual environment are mostly driven by these stereotyped activity contexts (Bruner, 1985) - e.g. mealtime or storytime - and that children learn most robustly in these contexts. On these accounts, children might become very sensitive to the co-occurrences between different activities (e.g., eating) and object categories (e.g., spoons, food). However, 

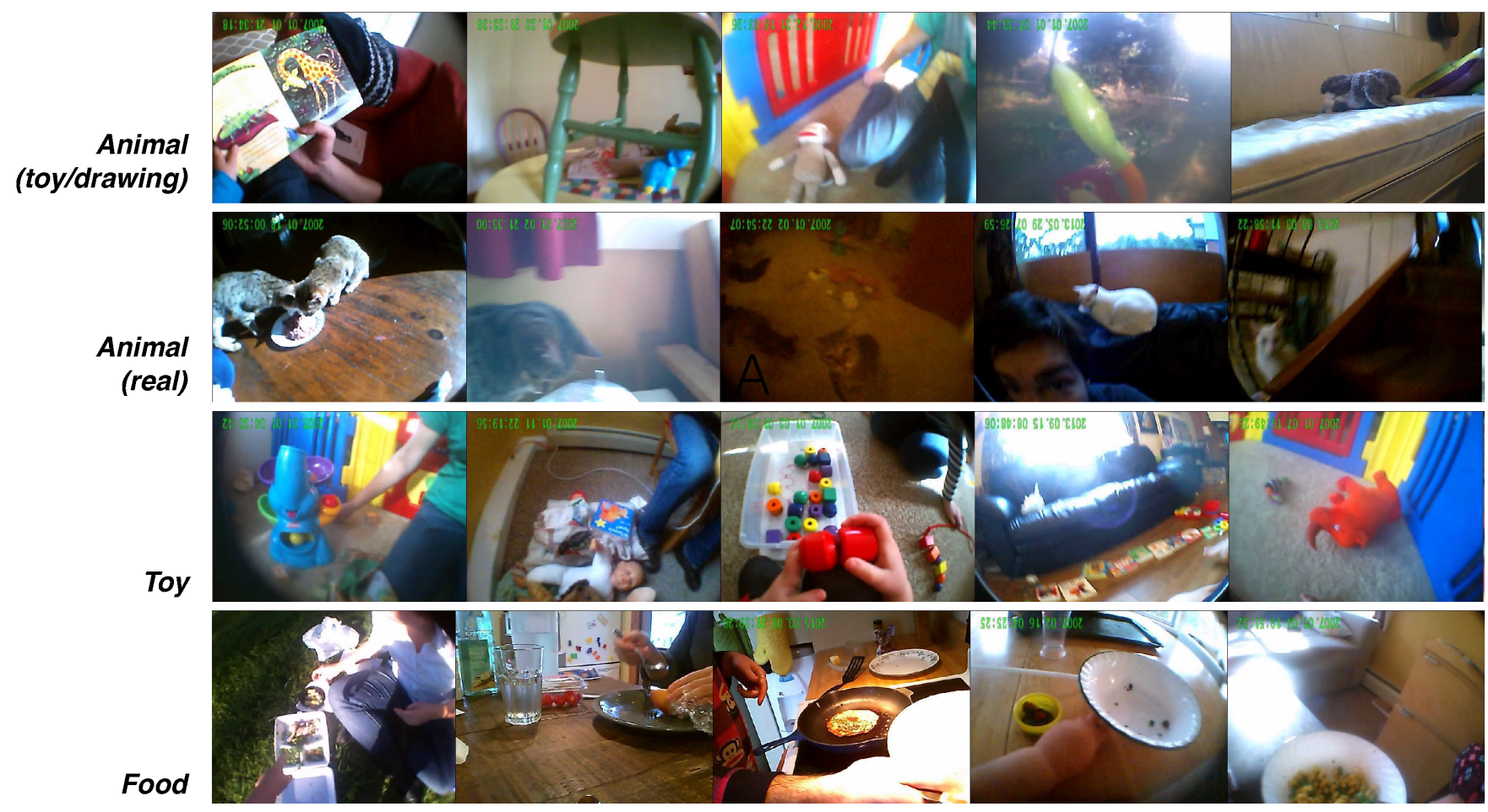

Figure 1: Example frames with annotations of four different categories.

no work has identified how consistently categories co-occur in natural environments. For example, while some activity contexts (e.g., storytime) lead to intuitive co-occurrences between object categories (e.g., between books and people), not all activity contexts will generate intuitive or consistent cooccurrences between object categories.

Finally, how infants interact with object categories will undoubtedly change what they learn about them. For example, children tend to generate informative views of objects while manipulating them - and, early in development, children's ability to sit and manipulate objects correlates with their perceptual abilities (Soska, Adolph, \& Johnson, 2010). Yet while most datasets used to train deep neural network models contain only photographs of object categories, many children - especially those in Western, industrialized cultures - will likely experience many categories through picture books as flat, stylized, 2D depictions. If children see very few real-life exemplars of a category relative to depictions (e.g., giraffes), this suggests that children must learn to generalize between these different visual formats in order to group these exemplars into one category. Further, this implies that these representations might be coarser than those experienced across many different formats. And if children only interact and manipulate a small set of categories - as suggested by Clerkin et al., 2017 - children may first learn about these frequently experienced categories and then use these representations to generalize to the categories they encounter very infrequently.

Here, we take a step towards answering these questions by characterizing the visual environment of two young children in a longitudinal corpus of head-mounted camera data (Sullivan, Mei, Perfors, Wojcik, \& Frank, 2020) from 6-32 months of age. To characterize trends in the visual environment over development, we collected annotations of several categories of objects (e.g., animals, vehicles, toys, food, furniture) present in the infant view, obtaining annotations on a randomly sampled set of 24,000 frames (i.e. around 59 frames per hour of recorded video). To provide a closer look into the kinds of objects children have the most intensive visual and haptic experience with, we also examined the specific objects that children interacted with during everyday activities. To do so, we annotated the basic-level identities (e.g., spoon, marker) of the objects that children were interacting with in the subset of frames where children's hands were visible.

\section{Method}

\section{Dataset}

The dataset is described in detail in Sullivan et al. (2020). Children wore Veho Muvi miniature cameras mounted on a custom camping headlamp harness ("headcams") at least twice weekly, for approximately one hour per recording session. One weekly session was on the same day each week at a roughly constant time of day, while the other(s) were chosen arbitrarily at the participating family's discretion. Videos captured by the headcam were $640 \times 480$ pixels, and a fisheye lens was attached to the camera to increase the field of view to approximately 109 degrees horizontal x 70 degrees verti- 


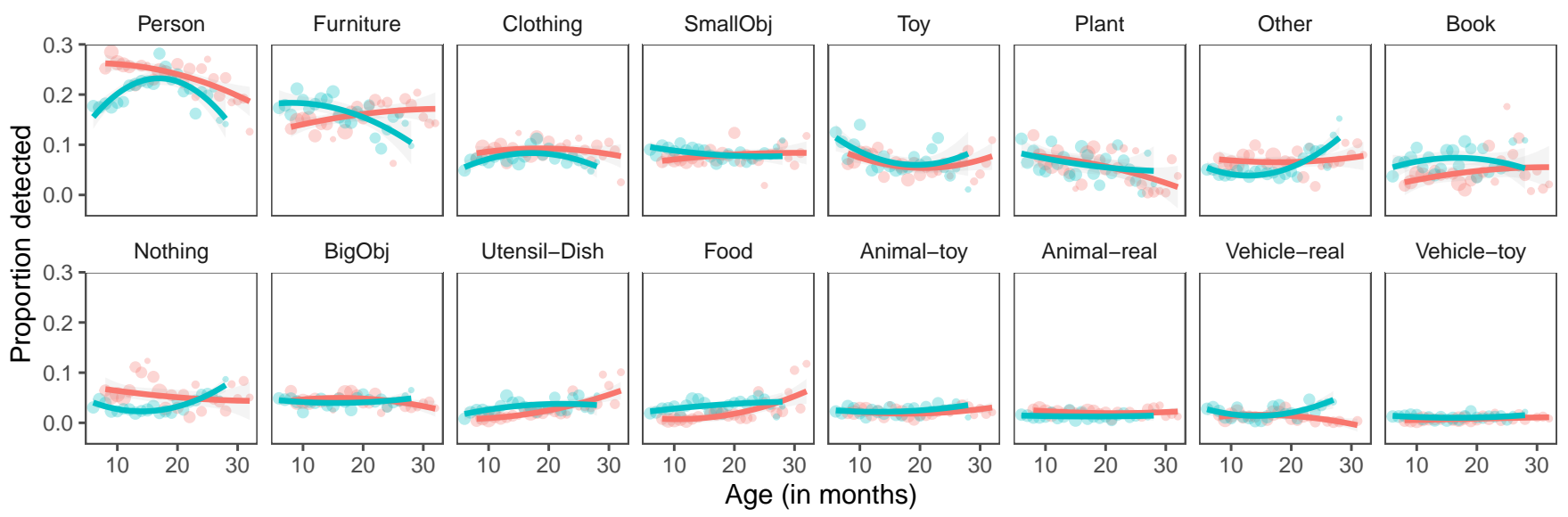

Figure 2: Frequency of categories annotated across the $24 \mathrm{~K}$ random frames plotted as a function of each child's age (in months); each child's age was calculated in days relative to the date that the videos were filmed and converted to months. Each color represents data from a different child.

cal. We randomly sampled 24,000 frames from videos of two of the children in the dataset $(\mathrm{S}, \mathrm{A})$ over the entire age range (6-32 months of age) At the time of recording, both children were in single-child households.

\section{Annotation procedures}

Categories in the infant view Annotations of the categories in the dataset were obtained using AWS Sagemaker; adult participants were recruited via Amazon Mechanical Turk. Participants viewed one image at a time and selected whether the following categories were present in the shown image: Animal (real), Animal (toy/drawing), Vehicle (real), Vehicle (toy/drawing), Plant, Clothing, Person, Furniture, Food, Utensil/Dish, Other Small Object, Other Big Object, Book, None of the above, or Nothing visible. We included Other Small Object and Other Big Object as categories that participants could use to indicate the presence of objects that fell outside of these categories but were still salient; additional instructions were provided to specify that Other Big Object refers to objects bigger than a chair, and that Other Small Object refers to objects small enough to be held with one or two hands (Konkle \& Caramazza, 2013). We chose this coding scheme after iteratively piloting options and examining frames ourselves. Two participants annotated each image, and were required to select at least one category before proceeding. Each category annotation in each image was assigned a confidence score (possible range: $0-1$, range in dataset: $0.5-1$ ) and individual annotations that had confidence scores below the 25th percentile were excluded from analyses (although all conclusions hold with and without these lowconfidence annotations).

We assessed the reliability of these annotations by comparing them to annotations made on the same task for a random subsample of 1200 frames on AWS Sagemaker, again using two participants per image ( $N=950$ frames after excluding low-confidence annotations). We found agreement was moderate (average Cohen's Kappa $=0.29$, but varied sub- stantially between different categories (range $=0.02,0.59$ ), as Cohen's Kappa is known to beharsh for sparse annotations. On average, there was a disagreement rate of $11.41 \%$ across categories between these two samples. Annotators disagreed most on whether Clothing was present in an image and whether Other Big Object was present (i.e. a big object that was not Furniture or a Vehicle). To assess the nature of these disagreements, we manually examined a random sample of 160 images with disagreements with $10 \mathrm{im}$ ages from each category. There were relatively equal proportions of images where annotations failed to identify a clear example of a category $(M=25 \%)$ or where annotations select an erroneous category label $(M=24 \%)$. However, we found that most $(M=50.62 \%)$ of the disagreements resulted from ambiguous exemplars, for example where the category was present but very distant, occluded, or blurry. Annotators also showed some disagreement about whether glossy photos of different categories in books should be counted as "real" or "toy/drawing," and whether partial views of people (i.e. child's own hands) should count as a Person. Going forward, we analyze the larger set of annotations with the caveat that there is inevitably some ambiguity in what counts as an exemplar of these categories (and that these data include both misses and false alarms). All annotations are openly available at the repository associated with this project (https://osf.io/ft4ka/).

Objects children interacted with We also annotated the objects that children were interacting with in a subset of these frames. To do so, we first selected the frames in which participants (recruited via Amazon Mechanical Turk) indicated that a child's hand or hands were visible in the image (see Long et al., 2020) and one author annotated 1817 of these frames, spanning 7 to 28 months of age with roughly equal proportions from the two children. The annotator noted what object the child was touching or pointing to within frames containing children's hands, using basic-level object categories such 


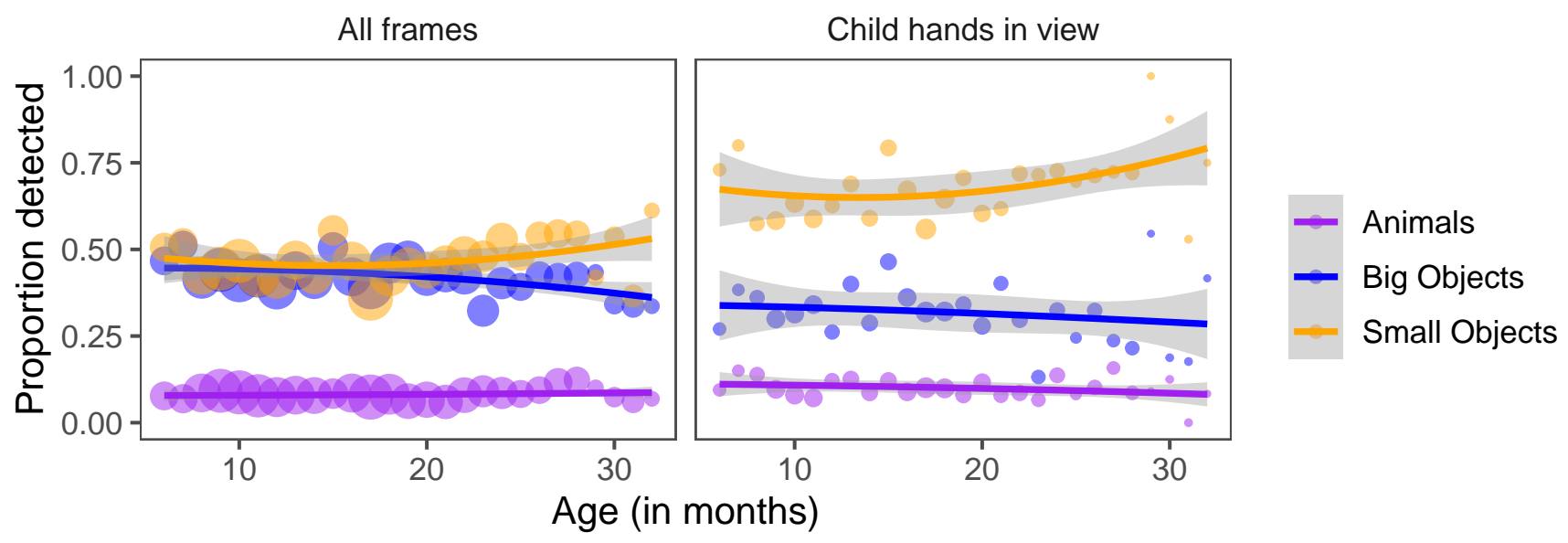

Figure 3: Frequency of animals (including toys) relative to big and small inanimate objects detected in the dataset, both when analyzing all frames that were annotated (left) and the subset of frames where a child's hand was visible in the frame (right).

as "block" and "cracker." If a child was holding a book and pointing to a depicted object in the book, the depicted object was noted as the category they were interacting with; otherwise, it was noted as Book. Food that was unidentifiable as a specific item (e.g., as crackers) was marked as "food," and baby toys that were unidentifiable as specific toys were marked "toy." When children were interacting with drawing or toy versions of different categories (e.g., a toy car), these annotations were marked with a '-drawing' and '-toy' modifier and counted as separate entries. If a view was allocentric, if there were no child hands in view, or if there were no objects that were visible, these frames were excluded from analysis; this left 1313 frames with annotations.

\section{Results}

\section{Which categories are prevalent in the child's view?}

First, we examined the overall prevalence of each category in the infant view. Somewhat surprisingly, we found that the prevalence of most of these categories were relatively stable both across the two children in the dataset as well as over developmental time (see Figure 2). This stands in contrast to prior work on the prevalence of faces/hands in the infant view (Fausey et al., 2016; Long et al., 2020), suggesting that these broader characteristics of children's visual experience may be more consistent.

We next examined the details of these environments. We found that people were by far the most prevalent of these categories: over $20 \%$ of the annotated frames contained people, far more than any other category (including all kinds of toys combined). In contrast, there were relatively few instances of animals in the infant view - either as toys or their reallife counterparts. Less than $5 \%$ of the frames contained any kind of depicted or real animal, and those frames contained depicted vs. real animals in equal proportion. Manual inspection of these frames containing animals revealed that the "real" animals had relatively little variety - they were overwhelmingly frames containing images of household pets (i.e., cats, dogs, and chickens, in the case of A), whereas the animals that were "toys/drawings" depicted a much larger variety of animals, as one might expect. Overall, these results suggest that - at least for these children - people are much more frequent than depictions or real-life versions of animals, indicating that toys and drawings may provide frequent input to their representations of these categories - despite the fact that animal names are often among children's first words (Frank, Braginsky, Yurovsky, \& Marchman, 2021) and often referenced in storybooks.

Far more prevalent than animals, instead, were objects. Views of furniture were the next most common category after people. However, in older age ranges, "big objects" - including Furniture, Vehicles, and Other Big Objects - tended to be less frequently in the view of infants than "small" objects - including Toys (of all kinds), Food, Utensils, Books, and Other Small Objects (see Figure 3). This effect was much exaggerated when we conducted this analysis on a subset of the frames where children's hands were also in view as a proxy for times when children were interacting with objects. In these frames, small objects tended to be much more prevalent in the frames that we annotated. These data are consistent with the idea that as children grow and become more adept at handling objects on their own, small objects may tend to be more often in view.

\section{Which categories co-occur in children's visual environment?}

Next, we examined the degree to which these categories appeared together in different frames. Figure 4 shows the cooccurrence of these categories, and reveals some relatively intuitive patterns that may reflect activity contexts. For example, Dishes and Food co-occurred quite frequently together, as did People and Clothing, and most Animals that were toys or drawings appeared when Books were also present. To determine which cells significantly deviate from chance we used a permutation analysis in which we shuffled the annotated 


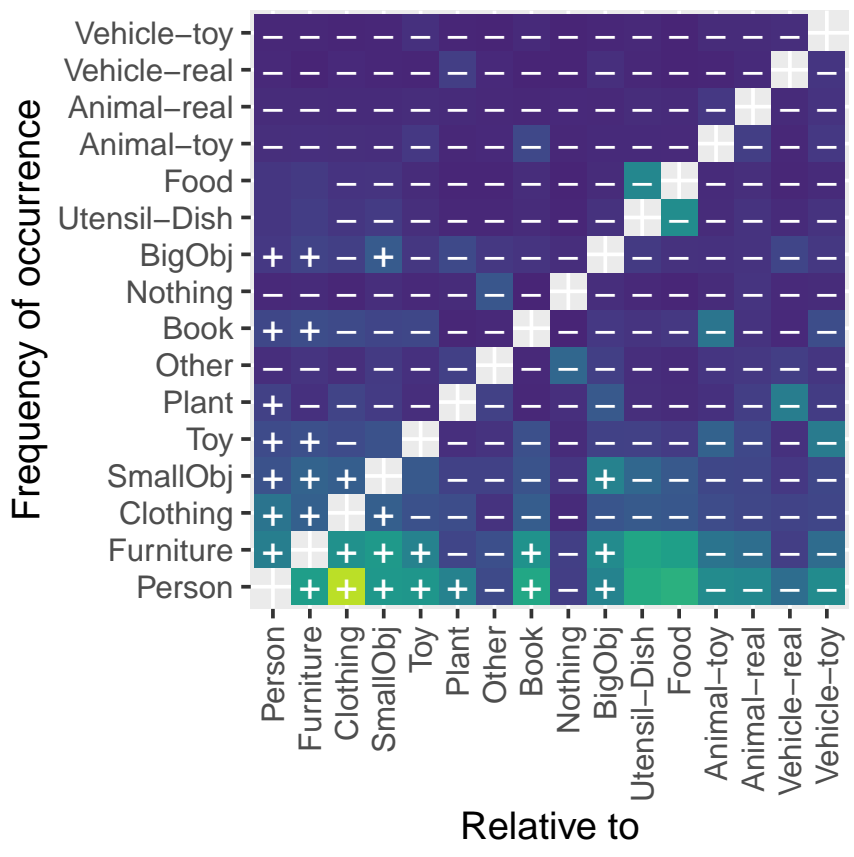

Figure 4: Co-occurrence between categories detected in the dataset. Each cell represents the probability that the category on the y-axis (e.g., clothing) occurs relative to the occurrence of the category on the x-axis (e.g., person). Lighter values indicate higher probabilities of co-occurrence $(\max =.8$, $\min =0$ ). Permutation analysis was used to determine which cell values were outside the $99 \%$ confidence intervals of counts: -: less than $99 \%,+$ : greater than $99 \%$.

category labels within each frame and examined the distribution of co-occurrences across 100 randomized co-occurrence matrices. The cells in the plot that occurred fewer times than expected by chance $(<99 \%$ of permuted cells) are labeled with a '-', while those that occurred more often than expected by chance ( $>99 \%$ of permuted cells) are labeled with a ' + '. These results suggest a strong co-occurrence structure rather than random occurrence, plausibly driven by activity contexts - such as playtime, mealtime, or storytime (Bruner, 1985).

\section{What objects do children tend to interact with?}

While many different categories may be in the child's view, not all of these objects may be experienced in the same way. In particular, it may be that children are more likely to form robust representations of objects that they physically interact with more often, and by extension they may also learn the labels of these objects earlier. In this analysis, we sought to analyze the basic-level identities of the objects that children tended to be interacting with in their home environments, and the distributions of those identities. While some work has found that the objects in view during mealtime tend to have a long-tailed Zipfian distribution (Clerkin et al., 2017), it is not yet known whether this finding will extend to objects that do not appear during mealtime and that children interact with during a wide range of activities. For example, there may

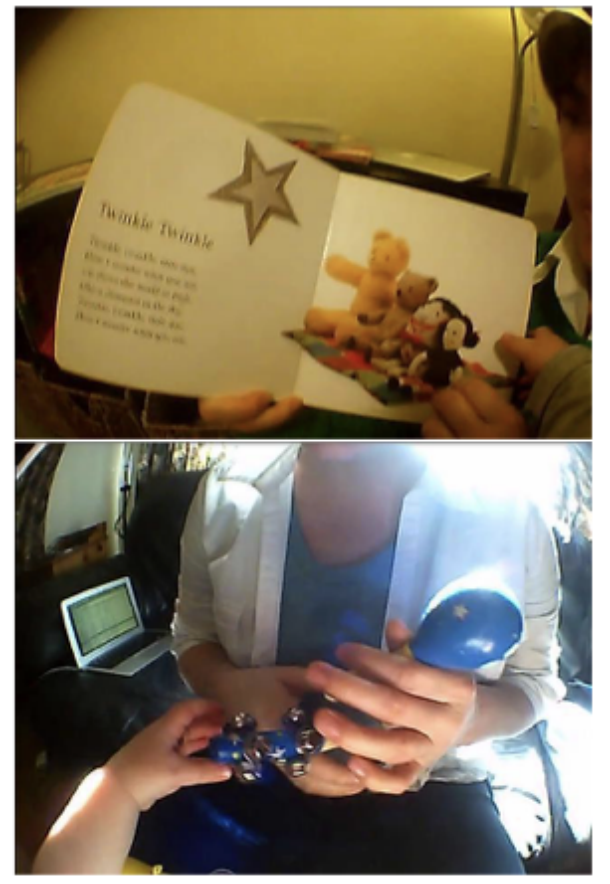

Figure 5: Example frames where we annotated the basic-level categories of objects children interacted with; many of these frames contained books and generic toys.

be far fewer objects that are only interacted with a limited number of times vs. seen a limited number of times.

In the frames with objects that children were interacting with, we found 132 unique categories when collapsing across formats (i.e. drawings, toys, real-life), and 148 unique categories when exemplars were considered separately across formats. When we examined which categories were most frequent, we found that books were overwhelmingly the most present object in the views of these two children, comprising over $20 \%$ of the objects that these children were seen to be interacting with. Generic baby toys (that were unidentifiable to the authors as specific toys) were the next most prevalent object category, and children were often seen to be touching or holding on to their caregivers (see top 20 most frequent categories in Figure 6 and example frames in Figure 57. Further, these three categories - book, toy, and person - were consistently the top three most frequent when we examined data separately for each child and by age groups (6-12 months, 12-18 months, 18-24 months).

Importantly, we found that the distribution of the objects children were interacting with roughly followed a power law distribution, when we included separate categories for different formats $(\alpha=1.82$, Kolmogorov-Smirnov distance $D=$ $0.07)$, when we collapsed across them $(\alpha=1.8, D=0.07)$, or when we excluded book, toy, and person $(\alpha=1.94, D=$ 0.09); the small D-values in each case suggest that the data roughly follow a power-law distribution. Thus, overall these results confirm that the distribution of the objects that chil- 
dren interact with is highly skewed, generalizing the findings of Clerkin et al., 2017.

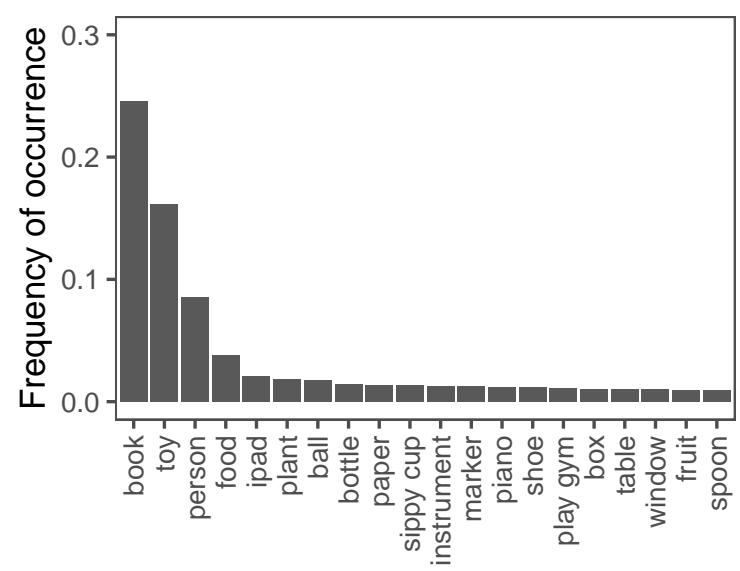

Figure 6: Top 20 most frequent categories that children's hands were interacting with in these egocentric videos.

\section{General Discussion}

What determines the categories that infants tend to see and interact with across early development? To examine the categories in the infant view, we analyzed a sample of random frames taken from a longitudinal dataset of two children (Sullivan et al., 2020). Overall, we found relative stability in these two children's visual environment over development, in contrast to prior work on the prevalence of social signals over this same developmental time period (Fausey et al., 2016; Long et al., 2020). The relative proportions of categories of objects (i.e., furniture, toys, animals, people) were consistent among the two individuals here, and across developmental time. People were most frequent, and a non-trivial proportion of frames didn't contain any discernible objects at all. However, these categories co-occured together in reliable patterns, revealing stereotypical combinations (i.e. utensil/dish and food, person and clothing) and suggesting that activity contexts, such as mealtime or storytime (Bruner, 1985) may structure the broad characteristics of young children's visual environment.

Yet while people were incredibly frequent in the child's view, animals - either as toys or their real-life versions - were relatively infrequent and occurred in equal proportions. This finding stands in contrast to a long literature documenting that even newborns prefer to attend to animate agents (Farroni et al., 2005), that visual cortex dedicates a remarkable amount of space to processing animals (Konkle \& Caramazza, 2013), and that animal names tend to be among children's firstlearned words (Frank et al., 2021). Therefore, children's heightened attention to animals (Farroni et al., 2005) likely interacts with frequency of occurrence in the visual field to drive early category learning.

Instead, we found that these children's viewpoints were likely to be dominated by small objects - such as food, books, or toys - especially when their own hands were present in the frame, suggesting that the statistics of children's visual environment shift substantially when they are acting on the world themselves. We also found that the distribution of these objects seem to follow a power-law distribution, as does word usage in natural language (Zipf, 1949). While mealtime has previously been used to characterize the objects in the infant view (Clerkin et al., 2017) and frames with food or utensil and dishes accounted for less than 5\% of views in the SAYcam dataset, we were unsure whether this also would be the case. However, the present analysis suggests that infants' interactions with different object categories may be Zipf-distributed, with most categories seen quite rarely, and a few categories dominating their experience.

If this result is generalizable, and children's visual experience is as skewed as their language experience, this distributional regularity provides strong constraints on the category and word learning mechanisms that can succeed in this environment (Hidaka, Torii, \& Kachergis, 2017; Lavi-Rotbain $\&$ Arnon, 2021). Although the Zipfian distribution of words can help learners segment speech (e.g., Kurumada, Meylan, \& Frank, 2013), it is theoretically challenging to learn wordreferent mappings from such skewed distributions both because the many rare referents are seen with such low frequency that learners may not have time to form strong mental representations and because the few frequent items may often co-occur and thus be difficult to disambiguate (Hidaka et al., 2017; Vogt, 2012). However, some word learning studies have found that Zipfian frequency distributions can improve learning (Hendrickson \& Perfors, 2019) and that adults can leverage knowledge of common objects to bootstrap the meanings of infrequent objects (Kachergis, Yu, \& Shiffrin, 2017). Indeed, the skewed distributions found here and in Clerkin et al. (2017) are dramatically different than the uniform distributions of categories fed to modern models of visual category learning - which nonetheless appear to mimic many aspects of the visual system (Jacob, Pramod, Katti, \& Arun, 2021). Future work that feeds computational models of category learning the same sequence of visual learning environments experienced by children across development may elucidate the set of online learning mechanisms needed to form robust representations from realistic visual inputs.

This work thus takes a first step in characterizing the categories in the visual environment over early development, calling for future work to understand the generalizability of these findings and their implications for models of early category learning. While we found consistent results across both age and the two children analyzed here, both children are from similar households, socioeconomic groups, and cultural contexts. Furthermore, these recordings were only taken when children were home with their parents (vs. at daycare), and these parents may have changed how they interacted with their children during these recording sessions. Variation across different households and communities will certainly change the objects that are frequently in the infant view: for example, picture books and toys are unlikely to be present in 
the views of many children in subsistence farming communities (Casillas, Brown, \& Levinson, 2020). Nonetheless, we predict that the distribution of objects that children interact will continue to follow a Zipfian distribution - regardless of which specific objects are most frequently experienced.

Of course, these results do not preclude the possibility that there are finer grained changes in how children experience object categories or changes the information that they encode. For example, the current analysis supports the intuition that toys and books are prevalent in the views of some infants, it does not document how children are interacting with these toys or what categories in the books their caregivers may be pointing out. More detailed coding schemes of the categories in the infant view could also yield divergent results. We anticipate that finer-grained analysis of the activities in naturalistic videos may uncover more subtle developmental trends.

Overall, this work highlights the need for systematic investigations of how the frequency of the categories in the child's view interacts with different attentional biases, learning mechanisms, and social cues to produce robust representations that support early category and language learning. An understanding of what is - and what is not - learnable solely from frequent exposures will provide constraints on our accounts of the learning mechanisms that allow children to learn so much so quickly. All data and code for these analyses are available at
\[ \text { https://osf.io/ft } 4 \mathrm{ka} / \]

\section{Acknowledgements}

We gratefully acknowledge the creators of the SAYCam dataset who made this work possible. This work was funded by a HAI Cloud Credit Grant to BL and MCF, a Jacobs Foundation Fellowship to MCF, a John Mereck Scholars award to MCF, and NSF \#1714726 to BLL.

\section{References}

Bruner, J. (1985). The role of interaction formats in language acquisition. In Language and social situations (pp. 31-46). Springer.

Casillas, M., Brown, P., \& Levinson, S. C. (2020). Early language experience in a papuan community. Journal of Child Language, 1-23.

Clerkin, E. M., Hart, E., Rehg, J. M., Yu, C., \& Smith, L. B. (2017). Real-world visual statistics and infants' firstlearned object names. Phil. Trans. R. Soc. B, 372(1711), 20160055.

Farroni, T., Johnson, M. H., Menon, E., Zulian, L., Faraguna, D., \& Csibra, G. (2005). Newborns' preference for face-relevant stimuli: Effects of contrast polarity. Proceedings of the National Academy of Sciences, 102(47), 1724517250.

Fausey, C. M., Jayaraman, S., \& Smith, L. B. (2016). From faces to hands: Changing visual input in the first two years. Cognition, 152, 101-107.
Franchak, J. M., Kretch, K. S., Soska, K. C., \& Adolph, K. E. (2011). Head-mounted eye tracking: A new method to describe infant looking. Child Development, 82(6), 17381750.

Frank, M. C., Braginsky, M., Yurovsky, D., \& Marchman, V. A. (2021). Variability and consistency in early language learning: The wordbank project.

Hendrickson, A. T., \& Perfors, A. (2019). Cross-situational learning in a zipfian environment. Cognition, 189, 11-22.

Hidaka, S., Torii, T., \& Kachergis, G. (2017). Quantifying the impact of active choice in word learning. In. Cognitive Science Society.

Jacob, G., Pramod, R., Katti, H., \& Arun, S. (2021). Qualitative similarities and differences in visual object representations between brains and deep networks. Nature Communications, 12(1), 1-14.

Kachergis, G., Yu, C., \& Shiffrin, R. M. (2017). A bootstrapping model of frequency and context effects in word learning. Cognitive Science, 41(3), 590-622.

Konkle, T., \& Caramazza, A. (2013). Tripartite organization of the ventral stream by animacy and object size. Journal of Neuroscience, 33(25), 10235-10242.

Kurumada, C., Meylan, S. C., \& Frank, M. C. (2013). Zipfian frequency distributions facilitate word segmentation in context. Cognition, 127(3), 439-453.

Lavi-Rotbain, O., \& Arnon, I. (2021). Visual statistical learning is facilitated in zipfian distributions. Cognition, 206, 104492.

Long, B., Kachergis, G., Agrawal, K., \& Frank, M. C. (2020). Detecting social information in a dense database of infants' natural visual experience. Https://Psyarxiv.com/Z7tdg/.

Sanchez, A., Long, B., Kraus, A. M., \& Frank, M. C. (2018). Postural developments modulate children's visual access to social information. In Proceedings of the 40th annual conference of the cognitive science society.

Soska, K. C., Adolph, K. E., \& Johnson, S. P. (2010). Systems in development: Motor skill acquisition facilitates three-dimensional object completion. Developmental Psychology, 46(1), 129.

Sullivan, J., Mei, M., Perfors, A., Wojcik, E., \& Frank, M. C. (2020). SAYCam: A large, longitudinal audiovisual dataset recorded from the infants perspective. PsyArXiv. Retrieved from https://psyarxiv.com/fy8zx/

Vogt, P. (2012). Exploring the robustness of cross-situational learning under zipfian distributions. Cognitive Science, 36(4), 726-739.

Yoshida, H., \& Smith, L. B. (2008). What's in view for toddlers? Using a head camera to study visual experience. Infancy, 13(3), 229-248.

Zipf, G. K. (1949). Human behavior and the principle of least effort. Addison-Wesley. 